\title{
Recent Advances in Intrusion Detection Systems: An Analytical Evaluation and Comparative Study
}

\author{
Ashish Kumar \\ School of Information \\ Technology, \\ MATS University, Raipur \\ (C.G.), India-492001
}

\author{
Shrikant Chandak \\ School of Information \\ Technology, \\ MATS University, Raipur \\ (C.G.), India-492001
}

\author{
Rita Dewanjee \\ School of Information \\ Technology, \\ MATS University, Raipur \\ (C.G.), India-492001
}

\begin{abstract}
As the emergence of computer, the use and effect of internet in our daily life is increased day by day. Security has become the greatest problem within and outside the organizations. User ID, passwords and firewalls are the common steps that organizations take to secure their computers. However, these are not so effective mediums in current context of unsecure eon. Intruders and attackers are so advanced that they access the computer and manipulate it, so they cannot be traced easily. Through this contribution objective is to find out \& present existing intrusion detection system (IDS) with their pros and cons that will be helpful to select the best one.
\end{abstract}

\section{General Terms}

IPS (Intrusion Prevention System), IDS (Intrusion Detection System), Denial-of-Service, Snort, Security.

\section{Keywords}

Network Security, Intrusion Detection, Intrusion Prevention, Social Engineerimg Toolkit.

\section{INTRODUCTION}

Day by day internet is growing and reached to everyone affects on many. People's dependencies are also increased with this. Most of our transaction are now shifted to the internet, thus it is also become a soft area for various types of security threats and attracts various types of attackers whose objective can be data stealing or data diddling, sniffing, password cracking or any other form of misuse of unauthorized data. So IDS can be the best solution for the above problem.

"Intrusion detection system plays a very crucial role in security of organization's information and data. It is a system that checks systems and network traffic and inspects that attacks originating from inside or outside the organization".[1].

These are the some selecting criteria of IDS

Objective/Aim Identification (Perform a risk/attack evaluation).

General Overview of available IDS.

Define the need of IDS precisely.

List out the IDS according to need or Purpose.

Cost Expense (Benefit) Analysis \& Selection of IDS.

Decide Working \& Policy (Implementation).

\section{EXPLORATORY STUDY ON} EXISTING IDS

Based on the study of various research contributions [2] [3] [4] [5], it has been found that around hundred IDS are available. Through this table some popular ones are presented here:

Table 1. Currently Available IDS: At a Glance

\begin{tabular}{|c|c|c|}
\hline AnaDisk & $\begin{array}{c}\text { AuditTrack for } \\
\text { Netware }\end{array}$ & Authd \\
\hline $\begin{array}{c}\text { AIDE } \\
\text { (Advanced } \\
\text { Intrusion } \\
\text { Detection } \\
\text { Environment) }\end{array}$ & AppShield & $\begin{array}{l}\text { BlackICE } \\
\text { Defender }\end{array}$ \\
\hline Bro & bv-LifeLine & $\begin{array}{l}\text { Check Point } \\
\text { RealSecure }\end{array}$ \\
\hline $\begin{array}{c}\text { Cisco Secure } \\
\text { IDS }\end{array}$ & Clog & $\begin{array}{l}\text { CMDS Computer } \\
\text { Misuse Detection } \\
\text { System }\end{array}$ \\
\hline $\begin{array}{l}\text { CRCMd5 Data } \\
\text { Validation Tool }\end{array}$ & $\begin{array}{l}\text { CyberCop } \\
\text { Monitor NT }\end{array}$ & $\begin{array}{c}\text { CyberCop } \\
\text { Monitor Solaris }\end{array}$ \\
\hline $\begin{array}{l}\text { CyberCop } \\
\text { Scanner }\end{array}$ & CyberCop Sting & DesktopSentry \\
\hline Detect IT & DiskSearch & Dragon Sensor \\
\hline $\begin{array}{l}\text { eTrust Internet } \\
\text { Defense }\end{array}$ & Extercept & $\begin{array}{c}\text { Exodus Cyber } \\
\text { Attack } \\
\text { Management } \\
\text { Service } \\
\end{array}$ \\
\hline $\begin{array}{c}\text { Firewall } \\
\text { Reporting Suite }\end{array}$ & FireStorm & ForensicToolkit \\
\hline Gabriel & $\begin{array}{l}\text { HP Openview } \\
\text { Node Sentry }\end{array}$ & HP-Tcpdump \\
\hline ICEcap & ICEpac & $\begin{array}{l}\text { iD2 Secure } \\
\text { Transport }\end{array}$ \\
\hline Ifstatus & Incident Manager & IP-Watcher \\
\hline $\begin{array}{l}\text { ISensor } \\
\text { Intrusion } \\
\text { Prevention }\end{array}$ & $\begin{array}{l}\text { Kane Security } \\
\text { Monitor }\end{array}$ & KaVaDo InterDo \\
\hline Klaxon & LSOF & $\begin{array}{c}\text { Lucent } \\
\text { RealSecure }\end{array}$ \\
\hline
\end{tabular}




\begin{tabular}{|c|c|c|}
\hline Man Hunt & NetDetector & $\begin{array}{c}\text { NetIQ Corp. } \\
\text { Security Manager }\end{array}$ \\
\hline Netlog & $\begin{array}{l}\text { Network Flight } \\
\text { Recorder }\end{array}$ & NetProwler \\
\hline neuSecure & OSSEC HIDS & OnlineGuardian \\
\hline Patriot IDS & $\begin{array}{l}\text { Prelude Hybrid } \\
\text { IDS }\end{array}$ & Peek \& Spy \\
\hline QRadar & RealSecure & Reactive IDS \\
\hline $\begin{array}{c}\text { Red Sire, } 1^{\text {st }} \\
\text { Watch Managed } \\
\text { IDS }\end{array}$ & Review & SafeBack \\
\hline Samhain & $\begin{array}{c}\text { Security } \\
\text { Management Pack } \\
\text { for MOM } 2000\end{array}$ & Security Manager \\
\hline Sentry & Shadow & SilentRunner \\
\hline SMARTWatch & Snort & Strata Guard \\
\hline StealthWatch & Suricata & Tcpdump \\
\hline Tcp_wrappers & Tklogger & Tocsin \\
\hline Tripwire & T-sight & TTY-Watcher \\
\hline $\begin{array}{l}\text { TurnKey } \\
\text { Network } \\
\text { Appliance }\end{array}$ & $\begin{array}{l}\text { Vanguard } \\
\text { Enforcer }\end{array}$ & $\begin{array}{l}\text { Verizon Federal } \\
\text { Network System } \\
\text { NetFacade }\end{array}$ \\
\hline ViewDisk & $\begin{array}{l}\text { Vigient Security } \\
\text { Agents }\end{array}$ & WinSNORT \\
\hline
\end{tabular}

Table 2. Comparison Table of some well-known IDS

\begin{tabular}{|c|c|c|c|c|c|}
\hline : & Bro & Snort & NFR & Suricata & $\begin{array}{c}\text { Dragon } \\
\text { Squire }\end{array}$ \\
\hline 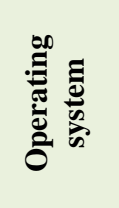 & 肴 & 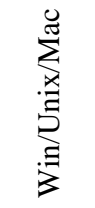 & 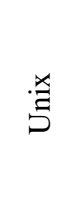 & 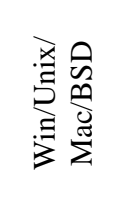 & 当 \\
\hline 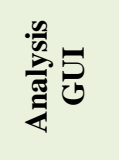 & $\tilde{\omega}$ & 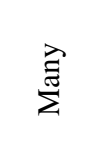 & 崩 & $\stackrel{\vec{\Xi}}{\stackrel{\Xi}{\Sigma}}$ & 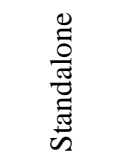 \\
\hline 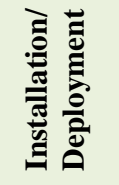 & 鸪 & 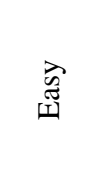 & 空 & 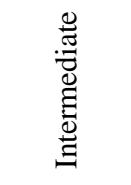 & 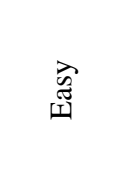 \\
\hline 宽 & $\stackrel{0}{\nu}$ & $\stackrel{\infty}{\infty}$ & $\stackrel{\infty}{\infty}$ & $\stackrel{\infty}{\nu}$ & 之 \\
\hline 啳 & $\stackrel{\circ}{Z}$ & $\stackrel{\infty}{\infty}$ & $\stackrel{\circ}{Z}$ & $\stackrel{\infty}{\infty}$ & $\stackrel{\circ}{z}$ \\
\hline 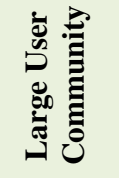 & $\stackrel{0}{z}$ & $\stackrel{\infty}{\infty}$ & 1 & 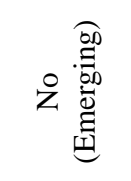 & 岕 \\
\hline कृष्ठ & 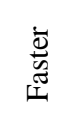 & 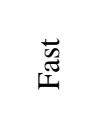 & $\frac{\Xi}{\Xi}$ & 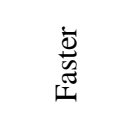 & 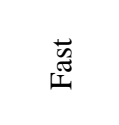 \\
\hline 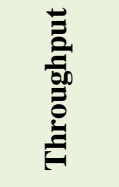 & 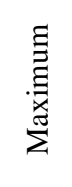 & $\begin{array}{l}\frac{\pi}{\pi} \\
\frac{0}{0} \\
\frac{0}{2}\end{array}$ & $\begin{array}{l}\frac{0}{\pi} \\
\frac{0}{0} \\
\frac{0}{2} \\
\Sigma\end{array}$ & 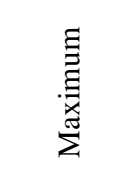 & 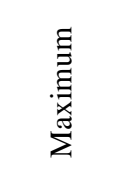 \\
\hline 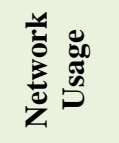 & 岕 & $\underset{\Xi}{\Xi}$ & 岕 & $\frac{n}{3}$ & $\frac{\Xi}{\Xi}$ \\
\hline لَّ & 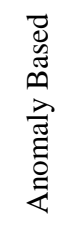 & 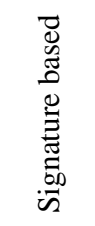 & 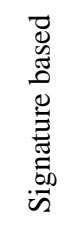 & 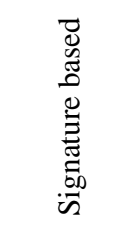 & 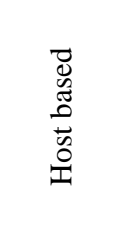 \\
\hline
\end{tabular}

Each IDS has specific design goals and objectives, thus having some specific/unique as well as some common features. From above listed IDSs, some well-known IDSs used by the organizations and now become de-facto standard as well, described here in brief with their features: 
These well-known IDSs with their conclusive highlights are as follows:

\subsection{Bro}

It is an anomaly based IDS, provides a real time network or pure traffic analysis that match the captured packets with desired rules applied by the user. It is used to analyze extensive logging functions related to application level details and to recording every connection observed on the wires. [6]

Highlights of Bro are as follows:

- Open-source.

- Specifically timely for scientific environments.

- Very useful in servers with default policies.

- $\quad$ Powerful on traded hardware.

- Intrusion prevention schemes are also supported.

The major benefits of Bro IDS are permitting it to measures from the desires of smaller institutions to those of the biggest research universities. Bro is implemented as a cluster set of conditions that uses three kinds of methods: Manager, Worker, and Proxy.

\subsection{Snort}

Snort has become the de-facto accepted for signature-based network IDS. It is based on the Libpcap library to capture packets. Snort is lightweight cross-platform network sniffing tool; it's evolved into a strong and full-featured IDPS. Snort engine allowed a single rule to be applied to any variation of a protocol. [7]

Some features of Snort are as follows:

- Can work on any operating system.

- Protocol examining capability.

- Condition examining capability.

- Packet reassembly capability.

- $\quad$ Lots of serving GUI's are available for analyzing the results.

Snort is configured using command line switches and optional Berkeley Packet Filter commands. Snort rules can be easily written by normal users, but powerful enough to detect a wide variety of hostile or merely suspicious network traffic. There are three basic actions that Snort can trigger when a packet matches a specified rule pattern: log, alert, or pass. [8]

\subsection{NFR}

Network Flight Recorder (NFR) gives the users a powerful tool to get the illegal attack in networks. With the efficiency of this tool, network administrator can know better about who is using or accessing their network and what their workers are doing. It is stand-alone configuration; a single NFR station gathers and stores information in a single instance.

Analysis in NFR is developed by script based language knows as $\mathrm{N}$-code, it uses web based interface to display the result with the use of java language. By its feature of not to interfere in network activity, this helps to analyze the standard or to free data from unwanted errors. Through the NFR one can store, retrieve, or archived the information to external drives [9] as well. However, this doesn't eliminate the requirement specialist to first analyze and categorize attack situations and system vulnerabilities, and hand-code the analogous rules and model or patterns in N-code for misuse detection. Due to the manual and ad hoc nature of the development process, NFR has low extensibility and adaptability. [10]

Highlights of the NFR are as follows:

- It provides built-in means for customization and extension.

- $\quad$ It is determined language, i.e., flexible and portable language for traffic exploration.

- It doesn't obstruct with network activity

- Having dynamic alerting function.

It works on UNIX based systems and is available in source code form. As it was previously available open source but now under proprietary license.

NFR is a multiuse network monitoring implement, operational for intrusion detection, usage exploration, and troubleshooting by system administrators. The construed $\mathrm{N}$-code language allows a user to write randomly complex scripts for examining incoming packets, in which NFR engine provide timing constrains to limit packets which helps to share system resources properly.

NFR comprises of a sum of modules, all responsible for a specific activity: packet suckers, a decision engine, back ends and a query interface. [11]

\subsection{Suricata}

An open source, high performance recent signature based network IDPS and Security Monitoring System for UNIX and Window based systems. It use PCAP recorder to log the traffic and provides offline analysis facility of PCAP files. [7][12]

Some Additional features are:

- $\quad$ Supports all operating system.

- $\quad$ Along with the IPS.

- Automatic detection of protocols with high performance.

- $\quad$ Network Security Monitoring (NSM).

- $\quad$ Filtering of alerts and events.

- Output format support many other tools to analyze data.

Suricata detects many anomalies in traffic it investigates. Having functionality of automatic detection of protocols on any port is very useful which helps to determine the malware and attacks.

Another progress in the Suricata engine is to employ native multi-threaded actions, something more necessary as network bandwidth increases. Suricata is planned from the start to take improvement of operating with multiple CPUs. A multithreaded discovery engine can make intelligent results on how to fragment processing and can match signature detection between these threads all within the same detection engine. [13]

\subsection{Dragon Squire}

The Dragon IDS products come across the experiments of inspecting over a modern network by providing high speed sensors to sense suspicious activity, allowed data to decide the impact of network attacks and scalability to set up and able to 
huge numbers of sensors, lacking negatively impacting the task of large networks. [14][15]

Dragon Squire is a host-based intrusion detection and firewall monitoring system that express at system logs for proof of malicious or suspicious submission activity, and monitors key system files for proof of damaging in real time. Dragon Squire has been tuned to prevent high load levels and minimize any negative system impact to a server's performance. Besides being an excellent system security tool, it also examines firewall logs, router actions and just about everything that can communicate SNMP or SYSLOG.

Some features of Dragon Squire are as follows:

- $\quad$ Ease of use because of single interface.

- Host \& network based versions.

- Clever to work over high-speed networks without dropping packets.

- $\quad$ Able to replay stored attacks for post-mortem and forensic analysis.

- $\quad$ Strong reporting capabilities.

- $\quad$ It has the ability to detect evasive IDS Techniques.

- $\quad$ GUI / command line alternative if preferred

- Capability of secure remote administration.

The Dragon Squire's signature archive contains suspicious events from a comprehensive range of operating systems. These actions check for guarding file transfers, failed login attempts, physical messages and system reboots. The library contains security messages such as Secure Shell, Qmail, Send mail and Apache Web servers.

It is used to observe network packets for proof of hacker and malicious worker activity by monitoring their system logs and firewall logs. It analyzes the system logs for proof of malicious or suspicious application programs in real time. It also observes main system records for evidence of tampering.
Being a tremendous system security tool, it can examine firewall logs, router events and reasonable about everything that can start SNMP or SYSLOG.

Dragon Squire has been engineered to have a slight influence on the servers it is protecting. It is also a superb supplement to the Dragon Sensor Network IDS.

\section{EXPERIMENTAL SCENARIO}

For experimental purpose a popular IDS, SNORT tool has been selected. Two hosts primarily connected to a LAN has been used, one become a victim machine and another becomes an attacker machine. SNORT, is installed on victim machine having Windows 8 and Intel's X86-based PC (64bits i5-2430M CPU@2.4GHz Processor with 2 GB RAM) configuration. Dummy attacker's machine having the same hardware configuration with BackTrack 5 R2 (a flavor of Linux) operating system [16]. All recorded data is contained in a local snort log file. The experiment was done to identify particular attacks and some packets. The traffic speed was low. With the use of Wireshark Packet Analyzer [17] the packets has been analyzed.

The three types of intrusions has been evaluated and examined for experimental purposes at here:

Attempt by a program to access the network resources.

Denial-of-service (e.g., Ping-of-death).

Unauthorized access from a remote machine (using Social Engineer Toolkit-SET [18]).

\section{EXPERIMENT RESULTS}

The generated $\log$ files are used to save the captured packets. The snort log file should be converted to ".csv" format for analysis in Wireshark. The log file of snort contains the following information, No., time, Source, Destination, Protocol, length, \& Info. Wireshark uses color coding to differentiate the packets received.

\subsection{Unauthorized Access by a Program to the Network Resources}

Fig 1: Attempt by a program to access the network resources 
Figure 1 shows the detail view of packets that are not trusted by the machine as the valid traffic in the network. Here a packet from the machine having IP address 10.203.45.2 from an unknown location in the network to the machine having IP address 224.0.0.251 is an illegal traffic.

\subsection{Denial of Service (Ping-of-death)}

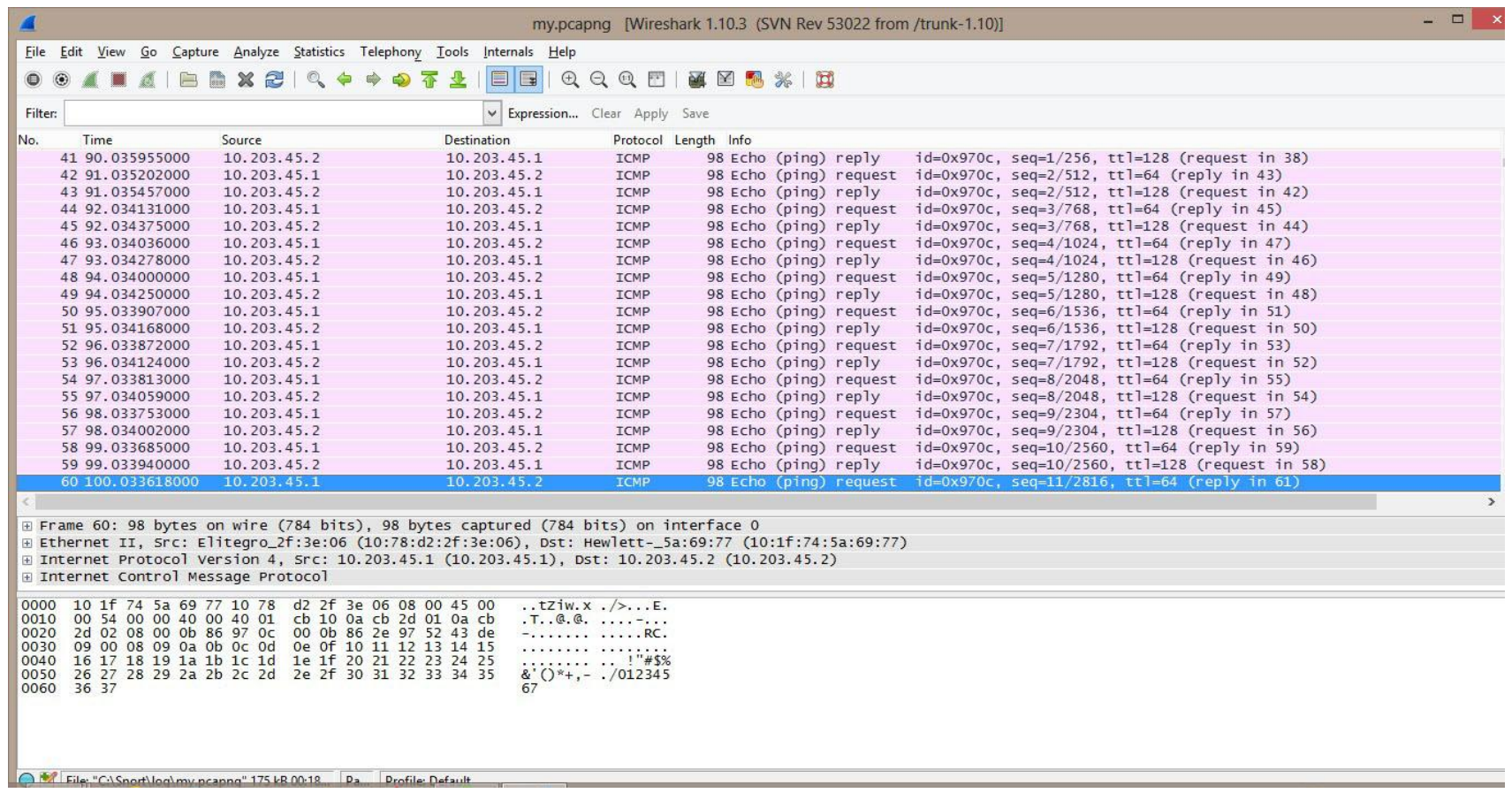

Fig 2: Denial-of-service (e.g., Ping-of-death)

In the figure 2, the ping of death attack is shown, that lead to Denial of Service. The machine having IP address 10.203.45.1 generated ping attack for the machine having IP address 10.203 .45 .2 .

\subsection{Social Engineering Attack (Cloning twitter.com)}

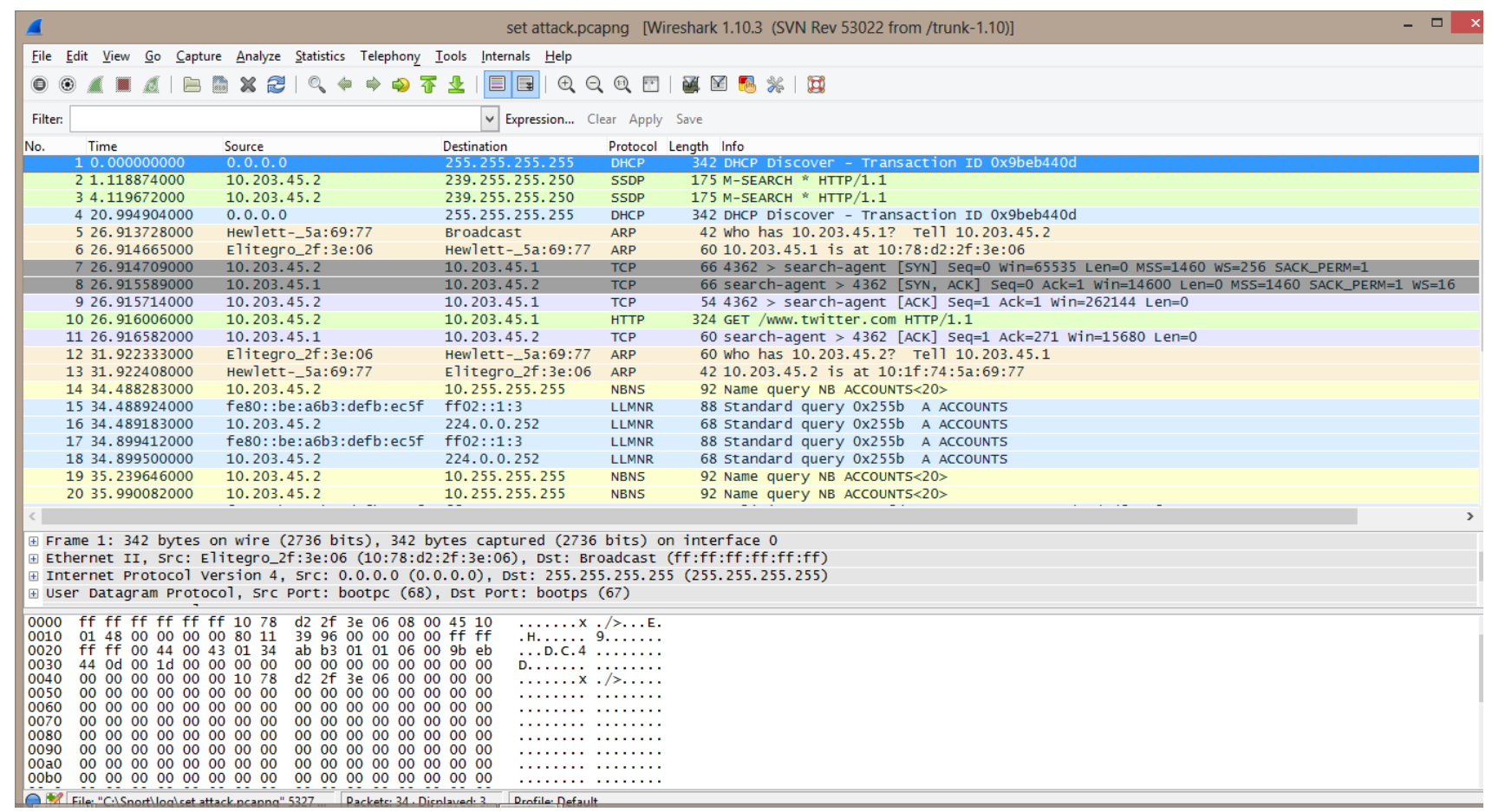

Fig 3: An Example of Social Engineering Attack (Cloning a social website twitter.com)

The social engineering attack is one of the advanced techniques used in the Metasploit framework to get a remote connection to the computer. The attackers utilize this attack to compromise the system for data theft by privilege escalation. 
If the attacker successfully runs privilege escalation he can get the administrator account. And the whole control of the system is transferred to the attacker who can use the system as the Trojan machine.

ReverseTCP connection was generated after trying to access twitter.com. It leads access to the machine having IP address 10.203.45.2 by using command line interface.

\section{DISCUSSION ON EXPERIMENTAL RESULTS}

The experiment shows how to configure SNORT and WireShark on Windows 8 machines. It is better to install Win Snort in place of Snort because it provides a GUI for better user interaction. The ReverseTCP successfully led the attacker to gain access to the system. It should have shown an alarm like a warning message on commad-line, with the details like the port number and type of protocol that is used to access the system, the destination and source IP addresses, with a particular color coding.

The packets also revealed unauthorized access by a program to the network resource when analyzed using WireShark.

The denial of service attack was captured and it showed continuous ping packets generated to the target machine.

The social engineering attack with the help of SET has been successfully executed. A social website twitter.com was cloned and run on the attacker's machine. When victim machine try to access the cloned website (i.e., twitter.com), it lets the backdoor to get into execute on the victim machine and generates a connection back to the attacker machine. The exploit used is aurora-internet explorer that allowed executing the payload on the victim machine.

\section{CONCLUSION}

There are many Open source IDS available for use, but Snort is the best alternative system to ensure network security. It is non GUI interface, takes time to get familiar. Through this paper, a detailed study on IDS has been performed and a popular IDS Snort has been implemented and configured on Windows-based environment. Some well known attacks has been executed and demonstrated successfully and the system was compromised. Snort was only used to capture the packets and WireShark analysis showed the illegal packets. Only detecting the attacks will not work, Snort should trigger some action that alerts the user about the attack. The GUI available for Snort should be comfortable for native users for configuration and running.

\section{REFERENCES}

[1] Fagg, C.R. 2001. Intrusion Detection Systems: Definition, Need and Challenges. White Paper. SANS Institute InfoSec Reading Room.

[2] Axelsson, S. March 2000. Intrusion Detection Systems: A Survey and Taxonomy. Technical Report. Chalmers University, Sweden.
[3] Sobirey, M. November 1999. Michael Sobirey's Intrusion Detection Systems page. http://wwwrnks.informatik.tu-cottbus.de/ sobirey/ids.html.

[4] Timberline Technologies LLC. Alphabetical List of Intrusion Detection Products. http://www.timberlinetechnologies.com/products/intrusio ndtct.html, [Last Updated 2009].

[5] Boyce, C.A.P. and Zincir-Heywood, A.N. October 2003. A Comparison of Four Intrusion Detection Systems for Secure E-Business. In Proceedings of the 6th International Conference on Electronic Commerce Research (ICECR03), ATSMA, IFIP, Dallas, USA.

[6] Varadarajan, G.K. October 2012. Web application attack analysis using bro ids. White Paper. SANS Institute InfoSec Reading Room.

[7] White, J.S. Fitzsimmonsb, T. and Matthews J. Quantitative Analysis of Intrusion Detection Systems: Snort and Suricata. In Proceedings of the SPIE, Cyber Sensing 2012

[8] Roesch, M. 1997. Snort - Lightweight Intrusion Detection for Networks. In Proceedings of the USENIX LISA'99 USA. November 1999.

[9] Inc. Network Flight Recorder. http://www.nfr.com, 1997

[10] Lee, W. Park, C. and Stolfo, S. 1999. Automated Intrusion Detection using NFR: Methods and Experiences. USENIX Intrusion Detection Workshop. California, USA.

[11] Undy, M. and Antonelli, C.J. 1998. Sifting the Network: Performing Packet Triage with NFR. CITI Technical Report 98-6. Center for Information Technology Integration, University of Michigan, USA.

[12] http://suricata-ids.org/features/all-features [last visited: $02 / 12 / 2013]$

[13] Albin, E. 2011. A Comparative analysis of the Snort and Suricata Intrusion Detection Systems. Master's Thesis, Naval Postgraduate School, Monterey, California, USA.

[14] Neumann, P. G. 1990. A Comparative Anatomy of Computer System/Network Anomaly Detection Systems, CSL, SRI BN-168, Menlo Park, CA, USA.

[15] Enterasys Networks. http://www.voxtechnologies.com/enterasys_files/pdf/ove rview-datasheet.pdf [last visited: 04/12/2013]

[16] BackTrack Linux. Available at: http://www.backtracklinux.org

[17] Wire Shark Packet Analyzer. Available at: http://www.wireshark.org

[18] Social-Engineer Toolkit (SET). Available at: https://www.trustedsec.com/ 